\title{
Fauna silvestre que afecta los cultivos en Boyacá y control del daño a cultivos de maíz
}

\section{Wildlife that affect plantations in Boyacá, and damage control on corn crops}

Fecha de recepción: 17 de abril de 2016

Fecha de aprobación: 20 de octubre de 2016
Israel Fernando Castillo-López ${ }^{1}$

Pablo Emilio Rodríguez-Africano ${ }^{2}$

Rubén Cornelio Montes-Pérez ${ }^{3}$

Diana Milena González-Valderrama ${ }^{4}$

\section{Resumen}

El trabajo tuvo como objetivos identificar las especies de fauna silvestre que afectan los cultivos en el Valle de Tenza y probar cuatro tratamientos no letales para proteger los cultivos de maíz. La identificación se efectuó durante cinco meses del año 2014. Se aplicaron 150 encuestas a campesinos de cinco municipios del Valle de Tenza por el método de bola de nieve, y se utilizaron 10 trampas Tomahawk y 30 Sherman, y cinco cámaras digitales de rastreo. La prueba de protección de los cultivos se efectuó por medio de la aplicación de cuatro tratamientos a base de repelentes, en el municipio de La Capilla, durante los meses de octubre de 2014 a enero de 2015, antes y durante la cosecha de maíz. Se aplicó un diseño completamente aleatorizado de los tratamientos y se evaluó el daño por análisis de varianza y contraste de medianas entre tratamientos.

Las entrevistas indicaron que 11 especies afectan los cultivos, principalmente ardillas (Sciurus granatensis) y faras (Didelphis marsupiales). Existen diferencias altamente significativas $(\mathrm{P}<0,01)$ entre la cantidad total de maíz dañado y sano, sin importar el tipo de tratamiento; sin embargo, no se encontró diferencia entre tratamientos $(P>0,05)$.

Palabras clave: ardilla; daño a cultivos; maíz; protección de cultivos; repelentes.

1 M. Sc. Universidad Pedagógica y Tecnológica de Colombia (Tunja-Boyacá, Colombia).

2 M. Sc. Universidad Pedagógica y Tecnológica de Colombia (Tunja-Boyacá, Colombia).

3 Ph.D. Universidad Autónoma de Yucatán (Mérida, México).mperez@correo.uady.mx.

4 M. Sc. Universidad Pedagógica y Tecnológica de Colombia (Tunja-Boyacá, Colombia). 


\begin{abstract}
The objective of this study was to identify the native fauna species that affect crops in the Valle de Tenza, and to test four non-lethal treatments to protect corn crops. The identification was carried out during five months of the year 2014. 150 surveys were applied to farmers from five municipalities in Valle de Tenza, following the "snow ball" method; additionally, 10 Tomahawk traps, 30 Sherman traps, and five digital tracking cameras were used. The test for crop protection was carried out by applying four treatments based on some repellents, in the municipality La Capilla, from October 2014 to January 2015, before and after harvesting the corn. A completely randomized treatment design was applied, and the damage was evaluated by analysis of variance and contrast of medians between treatments.

The interviews indicated that 11 species affect the crops, specially Sciurus granatensis and Didelphis marsupiales. There are significant differences $(\mathrm{P}<0.01)$ between the total amount of damaged and healthy corn, regardless the type of treatment used. Nevertheless, no differences were found between treatments $(P>0.05)$.
\end{abstract}

Keywords: corn; crop protection; crops damage; repellent; Squirrel.

\title{
Cómo citar este artículo:
}

Castillo-López IF, Rodríguez-Africano PE, Montes-Pérez RC, González-Valderrama DM. Fauna silvestre que afecta los cultivos en Boyacá y control del daño a cultivos de maíz. Rev. Cien. Agri. 2017; 14(1): 75-84. 


\section{Introducción}

En América existen numerosos reportes de aves y mamíferos que provocan daños a los cultivos; sin embargo, son escasos los estudios que han evaluado cuantitativamente las pérdidas ocasionadas por estos vertebrados a la agricultura (1). La apertura de nuevas áreas agrícolas y ganaderas donde existía vegetación nativa ha desencadenado un conflicto entre los humanos y la fauna silvestre, debido a la irrupción de poblaciones silvestres en los cultivos, así como en las ganaderías (2).

En varias zonas rurales de Colombia también hay pérdidas en diferentes cultivos agrícolas debido a vertebrados-plaga (3). Según declaraciones de los agricultores del Valle de Tenza ante la Corporación Autónoma Regional de Chivor (Corpochivor), mamíferos silvestres -especialmente ardillas (Sciurus granatensis)-, aves e insectos causan daños a diferentes cultivos, principalmente antes y durante la cosecha del maíz (Zea mays). Para disminuir los daños, los agricultores han empleado el control letal hacia las ardillas, sin considerar que estas cumplen varias funciones ecológicas destacadas en las comunidades naturales y en la cadena alimenticia del hábitat, como dispersoras de semillas, controladoras de insectos y presas de especies carnívoras (4). En este contexto surgió la necesidad de diseñar métodos de control no letal, dirigidos a la protección de los cultivos, y se encontró que los repelentes palatables, fisiológicos y olfatorios representan una alternativa de interés para la investigación (5). Así, los objetivos de esta investigación fueron identificar las especies de fauna silvestre que afectan cultivos en el Valle de Tenza y probar cuatro tratamientos no letales para controlar el daño a cultivos de maíz (Zea mays).

\section{Materiales y métodos}

\section{A. Métodos de identificación de especies de fauna silvestre que afectan cultivos}

Para el estudio fueron seleccionados los municipios de Garagoa, Pachavita, Tenza, La Capilla y Somondoco, debido a que sus agricultores han presentado quejas ante Corpochivor por el daño que la fauna silvestre ocasiona a sus cultivos. En estos municipios se aplicó una encuesta estructurada de 18 reactivos a 150 agricultores de 29 veredas seleccionadas. La selección del universo se realizó mediante el método no aleatorio denominado "Bola de Nieve" (6). Con el fin de aplicar la encuesta a los agricultores se seleccionaron al azar cinco o seis veredas en cada municipio, tal como se muestra en la Tabla I. Con base en los datos obtenidos de las encuestas se organizó la información de acuerdo con el porcentaje de afectación por cada cultivo. Los daños a los cultivos que reportan las personas encuestadas son consumo de los frutos y marcas en los frutos o partes de las plantas; además, informan de avistamientos de las especies de fauna silvestre consumiendo los frutos directamente.

Tabla I. Veredas seleccionadas en cada uno de los municipios objeto de estudio, donde se aplicaron las encuestas.

\begin{tabular}{|c|c|c|c|c|c|}
\hline Municipio & Garagoa & Pachavita* & La Capilla & Tenza & Somondoco \\
\hline V & $\begin{array}{c}\text { Bancos de } \\
\text { Páramo }\end{array}$ & Aguaquiña & Camagoa & Barzal & Boya 1 \\
\hline \multirow{2}{*}{$\begin{array}{l}\text { e } \\
r\end{array}$} & Caracol & Buenavista & Chaguatoque & Cora Chiquito & Cabrera \\
\hline & Fumbaque & Hatogrande & Hato & Quebradas & Guaduas \\
\hline e & Hipaquira & Pie de Peña & Palma Abajo & Resguardo & Pancupa \\
\hline d & Maciegal & Soaquira & Peña & Rucha & Richa \\
\hline $\begin{array}{l}\mathrm{a} \\
\mathrm{s}\end{array}$ & $\begin{array}{c}\text { Resguardo } \\
\text { Abajo }\end{array}$ & & Ubaneca & Volcán & Zarzal \\
\hline
\end{tabular}


Posteriormente, siguiendo los resultados obtenidos de las encuestas, se seleccionó la vereda de cada municipio que registró la mayor cantidad de afectación a los cultivos por la fauna silvestre, con el fin de realizar la captura de las especies reportadas. Se efectuó la instalación de cinco cámaras digitales de rastreo Bushnell ${ }^{\circledR}$ 6MP Ref. X119327 8-C, 10 trampas Tomahawk ${ }^{\circledR}$ y 30 Sherman $^{\circledR}$, en fincas ubicadas en las veredas seleccionadas. Las capturas se realizaron durante los meses de julio a noviembre del año 2014, que es la época de cosecha de maíz en la zona. Las trampas se ubicaron en tres diferentes estratos alto $(5,1 \mathrm{~m}-10 \mathrm{~m})$, medio $(5,0$ $\mathrm{m}-1,0 \mathrm{~m})$ y bajo $(<1,0 \mathrm{~m})$, con diferentes coberturas vegetales y camufladas con material vegetal, $y$ se desactivaron durante tres días, con el fin de que las especies de fauna silvestre se acostumbraran al ingreso a las trampas para consumir el cebo (bolas de avena con frutos secos, esencia de plátano o mantequilla de maní); al tercer día fueron activadas para capturarlas. Las trampas permanecieron ocho días en cada una de las veredas seleccionadas, y fueron revisadas diariamente para registrar las capturas de ejemplares, en horario de las 6:30 a las 14:00 horas. El periodo total de captura fue de 60 días (inicio de julio a finales de septiembre del año 2014). A partir de estas acciones se procedió a identificar los ejemplares capturados, mediante guías de campo (7).

\section{B. Métodos para controlar el daño a cultivos de maíz}

Se seleccionó el municipio de La Capilla para la evaluación de daño al cultivo de maíz (Z. mays) en parcelas sometidas a tratamiento de protección. Se eligieron dos cultivos en precosecha, divididos en ocho parcelas de 20×10 m cada una y con $3 \mathrm{~m}$ de distancia entre ellas; se delimitaron por medio de una barrera física (granel) y el callejón de $3 \mathrm{~m}$ de ancho correspondiente a la distancia entre parcelas. Se aplicaron cuatro tratamientos (Tabla II), siguiendo un diseño experimental completamente aleatorizado (Tabla III).

Tabla II. Tratamientos aplicados en los dos cultivos de maíz en el municipio de La Capilla.

\begin{tabular}{|c|l|}
\hline Nombre & \multicolumn{1}{|c|}{ Preparación } \\
\hline Tratamiento 1 (T 1) & $\begin{array}{l}\text { Biopreparado con ají (repelente palatable), se pulverizaron 200 g de } \\
\text { semillas de ají (Capsicum spp.) y se suspendieron en 10 L de agua; se } \\
\text { añadió un pegante que se elaboró mezclando 1 L de Coca-Cola }{ }^{\circledR} \text { en } \\
\text { 20 L de agua y se dejó reposar 15 minutos; esta mezcla se aplicó sobre } \\
\text { mazorcas con fumigadora (8). }\end{array}$ \\
\hline Tratamiento 2 (T 2) & $\begin{array}{l}\text { Creolina (repelente olfatorio); se rodearon las parcelas con troncos y se } \\
\text { les aplicó una mezcla de 3 ml de creolina en 1 L de agua (Juan Cardozo, } \\
\text { comunicación personal). }\end{array}$ \\
\hline Tratamiento 3 (T 3) & Mixto (T 1 y T 2) \\
\hline Tratamiento 4 (T 4) & Testigo, sin repelente. \\
\hline
\end{tabular}

La evaluación del daño se realizó durante cuatro meses (octubre del 2014 a enero del 2015), antes y durante la cosecha, llevando un seguimiento semanal de los tratamientos. Al finalizar la cosecha se determinó la cantidad de maíz dañado, identificado por presentar exposición y marcas del consumo de granos de la mazorca, y de maíz sano, identificado por no presentar alteraciones en la estructura de la mazorca. Los resultados se presentan tanto en peso seco de mazorca y número de mazorcas, y fueron contrastados por análisis de varianza de una sola vía o comparación de medianas (9). 
Tabla III. Distribución de los tratamientos en los dos cultivos de maíz en el municipio de La Capilla.

\begin{tabular}{|c|c|c|}
\hline \multirow{4}{*}{ Cultivo 1 } & Parcela & Tratamiento \\
\cline { 2 - 3 } & 1 & Mixto T 3 \\
\cline { 2 - 3 } & 2 & Ají T 1 \\
\cline { 2 - 3 } & 3 & Creolina T 2 \\
\cline { 2 - 3 } & 4 & Creolina T 2 \\
\cline { 2 - 3 } & 5 & Control T 4 \\
\hline \multirow{4}{*}{ Cultivo 2 } & 6 & Control T 4 \\
\cline { 2 - 3 } & 7 & Ají T 1 \\
\cline { 2 - 3 } & 8 & Mixto T 3 \\
\hline
\end{tabular}

\section{Resultados y discusión}

\section{A. Especies identificadas de fauna silvestre que afectan cultivos}

Las encuestas hacen referencia a reportes históricos acumulados de los agricultores, quienes mencionan cinco especies de mamíferos y seis de aves que afectan los cultivos. La Figura 1 muestra las especies que afectan cultivos en las veredas objeto de estudio, de acuerdo con las encuestas, cantidad de fotografías y capturas.

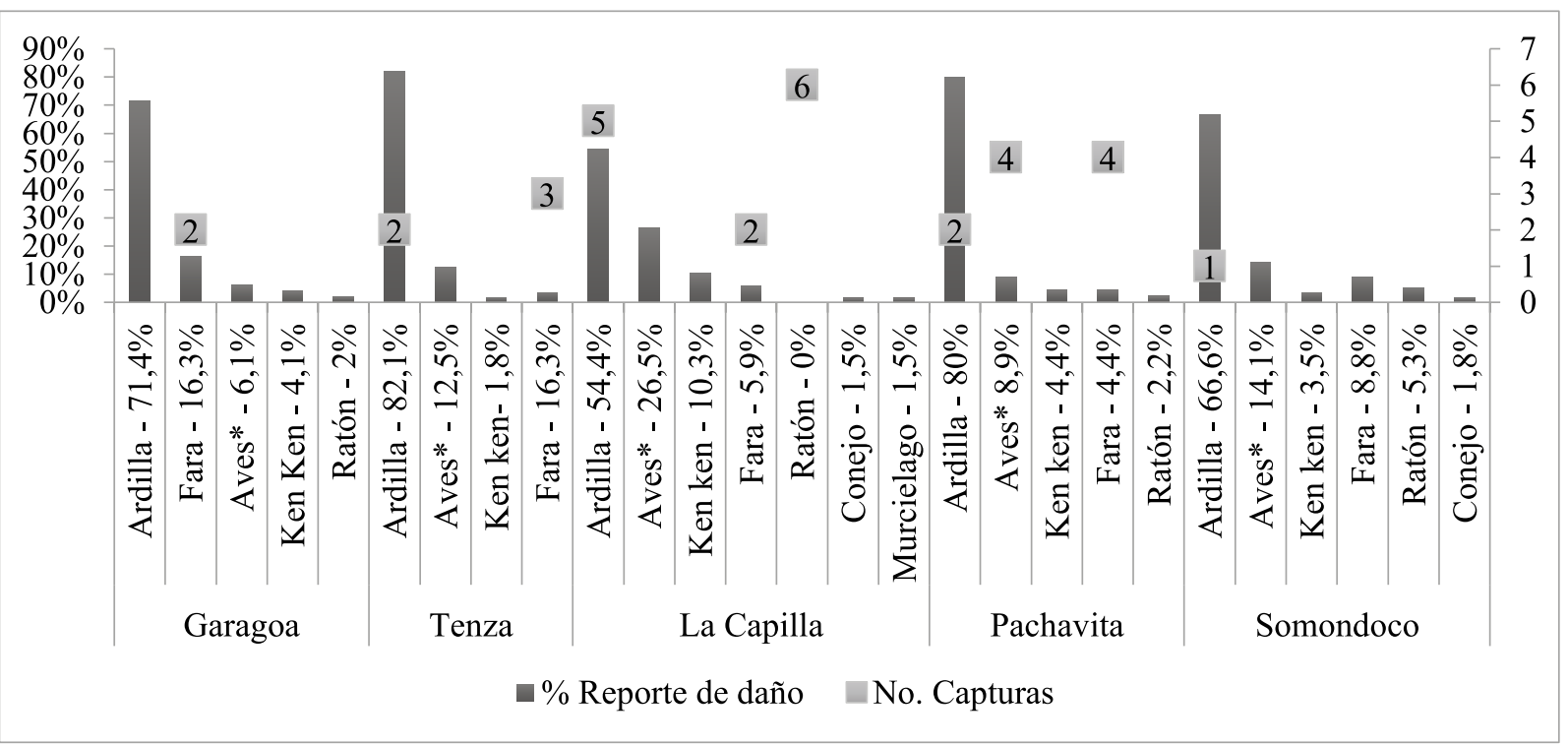

Fig. 1. Especies de fauna silvestre y cantidad de ejemplares que afectan cultivos en las veredas objeto de estudio, de acuerdo con porcentaje de daño reportado por encuestas y captura en trampas y fotografías.

*Especies de aves: chafi (Icterus chrysater), mirla negra (Turdus fucaster), mirla blanca (Mimus gilvus), babagui (Phaetucticus aureoventris) y azulejo (Thraupis episcopus).

Las principales especies de fauna silvestre involucradas en el conflicto son ardilla (Sciurus granatensis), ratón (Chilomys instans), fara (Didelphis marsupialis) y diferentes especies de aves: mirla blanca (Mimus gilvus), mirla negra (Turdus fucaster), chafi (Icterus chrysater), azulejo (Thraupis episcopus) y el ken ken (Cyanocorax yncas); otro daño fue por gorgojo (Sitophilus zeamais) y gusano del maíz (Spodoptera frugiperda). En la Figura 2 se muestran las especies de fauna silvestre involucradas en el daño y el número de cultivos que afectan, según las encuestas. En la Tabla IV se muestran 36 cultivos afectados por la fauna silvestre. 


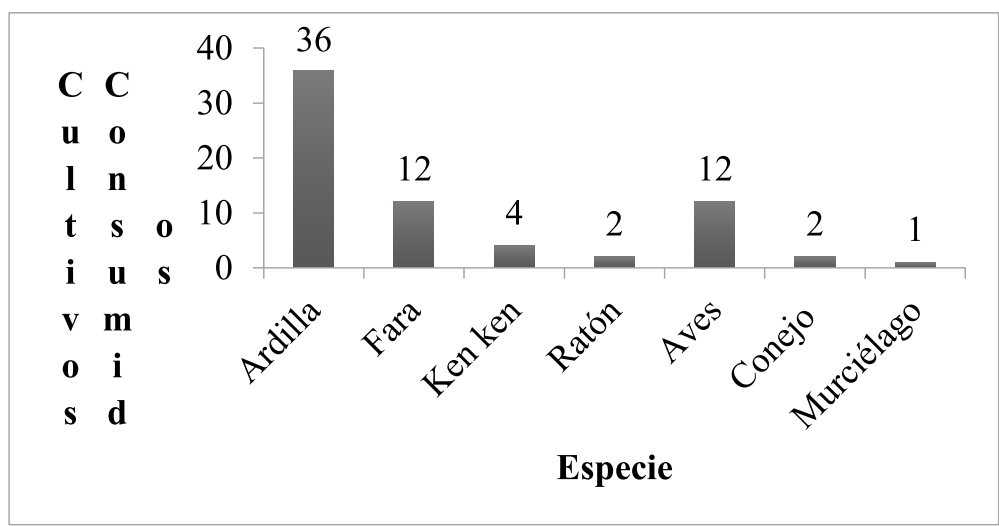

Fig. 2. Fauna silvestre y número de cultivos que afecta, según las encuestas.

Tabla IV. Cultivos y porcentaje de afectación ocasionada por fauna silvestre en los municipios evaluados, de acuerdo con encuestas.

\begin{tabular}{|l|r|l|r|l|r|}
\hline Maíz & $21,2 \%$ & Durazno & $1,9 \%$ & Mora & $0,7 \%$ \\
\hline Plátano & $11,2 \%$ & Fríjol & $1,9 \%$ & Mamey & $0,7 \%$ \\
\hline Guayaba & $7,8 \%$ & Maracuyá & $1,5 \%$ & Yuca & $0,7 \%$ \\
\hline Aguacate & $7,1 \%$ & Café & $1,5 \%$ & Tomate chonto & $0,7 \%$ \\
\hline Arveja & $5,9 \%$ & Manzana & $1,5 \%$ & Ciruela & $0,4 \%$ \\
\hline Pepino & $4,4 \%$ & Pera & $1,5 \%$ & Piña & $0,4 \%$ \\
\hline Tomate de árbol & $4,1 \%$ & Curuba & $1,5 \%$ & Granadilla & $0,4 \%$ \\
\hline Mango & $3,7 \%$ & Pimentón & $1,5 \%$ & Papa & $0,4 \%$ \\
\hline Guatila & $3,4 \%$ & Habichuela & $1,5 \%$ & Haba & $0,4 \%$ \\
\hline Naranja & $2,9 \%$ & Chamba & $1,1 \%$ & Cidrón & $0,4 \%$ \\
\hline Mandarina & $2,9 \%$ & Papaya & $1,1 \%$ & Habano & $0,4 \%$ \\
\hline Chirimoya & $2,2 \%$ & Feijoa & $0,7 \%$ & Pepino cohombro & $0,4 \%$ \\
\hline
\end{tabular}

* El daño a los cultivos se da basados en el criterio de los encuestados.

\section{B. Evaluación de los métodos de control de daño a cultivos de maíz}

La Tabla $V$ muestra las cantidades en peso y número de maíz dañado y sano por la ardilla (S. granatensis) y otras especies, en las ocho parcelas que fueron sometidas a los tratamientos. La Figura 4 muestra las medianas y cuartiles de cantidad de maíz sano y dañado en todos los tratamientos en unidad de masa y cantidad de mazorcas. La prueba de Mann-Whitney mostró $(\mathrm{W}=0,0)$ que existe una diferencia altamente significativa entre el número y la masa de maíz dañado por fauna silvestre y la masa y número de maíz sano $(P<0,01)$. 
Tabla V. Resultados en cultivos de maíz (Z. mays) sometidos a los tratamientos.

\begin{tabular}{|c|c|c|c|c|c|c|c|c|}
\hline Parcela & Tto. & \# Maíz sano & $\begin{array}{c}\text { \# Maíz } \\
\text { dañado } \\
\text { por } \\
\text { ardilla }\end{array}$ & $\begin{array}{c}\text { \# Maíz } \\
\text { dañado por } \\
\text { otros }\end{array}$ & $\begin{array}{c}\text { Maíz } \\
\text { sano } \\
\mathbf{( k g})\end{array}$ & $\begin{array}{c}\text { Maíz } \\
\text { dañado } \\
\text { por } \\
\text { ardillas } \\
\mathbf{( k g})\end{array}$ & $\begin{array}{c}\text { Maíz } \\
\text { dañado } \\
\text { por otros } \\
\text { (kg) }\end{array}$ & $\begin{array}{c}\text { Peso } \\
\text { total } \\
\text { maíz }\end{array}$ \\
\hline 1 & 3 & 138 & 2 & 11 & 19 & 0,026 & 0,2 & 19,21 \\
\hline 2 & 1 & 131 & 2 & 4 & 13,8 & 0,028 & 0,06 & 13,8 \\
\hline 3 & 2 & 166 & 1 & 9 & 24,8 & 0,012 & 0,2 & 25 \\
\hline 4 & 2 & 104 & 0 & 2 & 15 & 0 & 0,015 & 15,015 \\
\hline 5 & 4 & 51 & 0 & 12 & 8 & 0 & 0,168 & 8,168 \\
\hline 6 & 4 & 145 & 22 & 3 & 18 & 3 & 0,042 & 21,042 \\
\hline 7 & 1 & 153 & 7 & 1 & 20 & 1 & 0,012 & 21,012 \\
\hline 8 & 3 & 156 & 14 & 1 & 20 & 2 & 0,015 & 22,015 \\
\hline
\end{tabular}
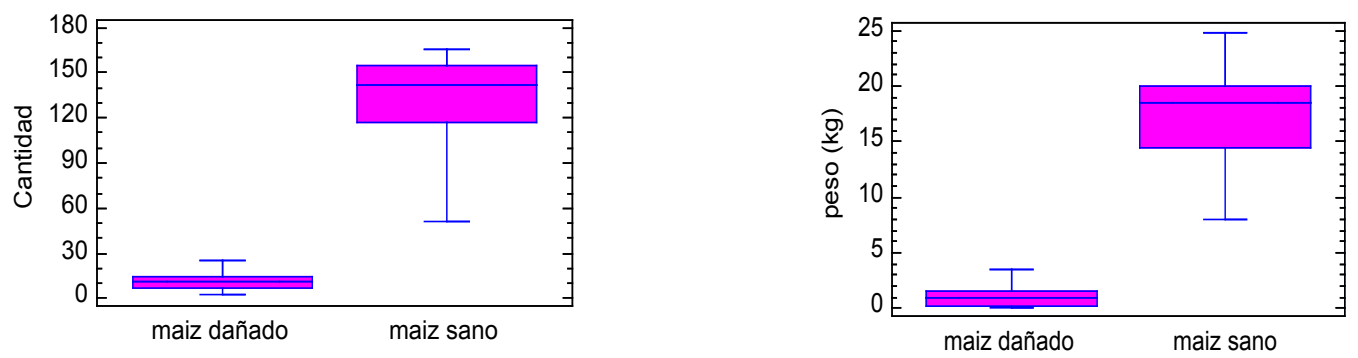

Fig. 4. Gráficas de cajas y bigotes para peso $(\mathrm{kg})$ y cantidad de maíz sano y dañado totales de los tratamientos, por fauna silvestre en los dos cultivos del municipio de La Capilla.

La Figura 5 presenta los intervalos de confianza al $95 \%$ de maíz dañado en cada tratamiento. El análisis de varianza no mostró diferencias signifi- cativas $(P>0,05)$ en la cantidad de maíz dañado entre tratamientos, pero sí una ligera tendencia al aumento de maíz dañado en el grupo testigo. 


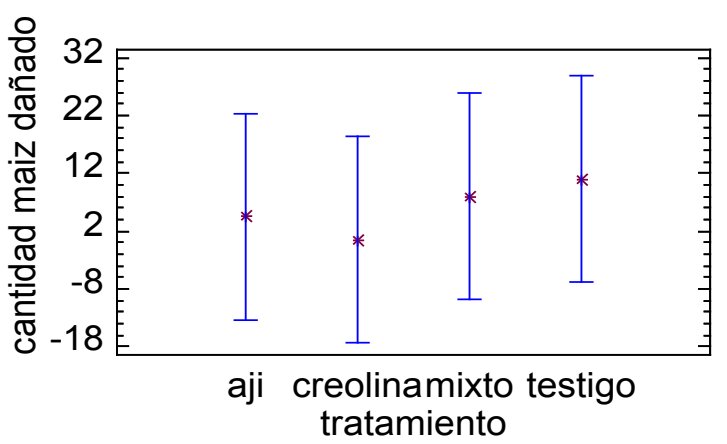

Fig. 5. Intervalos de confianza al $95 \%$ de las medias, en cantidad de maíz dañado por fauna silvestre, entre los tratamientos en el municipio de La Capilla.

El mayor porcentaje de daños en los cultivos de los municipios evaluados se presenta por el ataque de pequeños mamíferos, lo cual concuerda con Lamarque et al. (10), quienes reportan que "en términos de la escala de impacto en el ser humano, los pequeños mamíferos, los cuales se reproducen en grandes cantidades, son los que realizan el mayor impacto". El tipo de uso del suelo que se ha realizado en los municipios objeto de estudio ha ocasionado una fragmentación importante de los bosques nativos, lo que puede ser una de las razones del ataque de la fauna silvestre a los cultivos.

El cultivo con mayor afectación es el maíz ( $Z$. mays), con un $21,2 \%$, teniendo en cuenta que tiene mayores áreas de siembra en todos los municipios; esta información se confirmó durante la recolección de los datos en los predios visitados. Los cultivos menos afectados $(<1,1 \%)$ y otros más tienen menor extensión sembrada; además, los cultivos son, en su mayoría, minifundistas, dedicados al autoconsumo y sin tecnificar, por lo tanto, el daño que se genere a la pequeña producción de alimentos tiene alto impacto a la economía de subsistencia del productor (11).

En Colombia, los reportes sobre las especies que afectan cultivos y sobre métodos de control son nulos; en contraste, en Chiapas, México, RomeroBalderas et al. (1) mencionan que se dispone de tres reportes de las especies de aves y mamíferos consideradas nocivas por los agricultores locales.

De acuerdo con las encuestas, la ardilla (S. granatensis) es la especie dominante en cuanto al daño a los cultivos, entre estos el maíz (Z. mays); sin embargo, se evidenciaron daños a los diferentes tipos de cultivos y se comprobó la existencia del conflicto humano-fauna silvestre; se recomienda aumentar el esfuerzo de muestreo y valorar económicamente el daño en términos de beneficios sociales. Los rendimientos de maíz (Z. mays) en el Valle de Tenza serían normalmente bajos, debido a que son producciones en minifundios $y$, por tanto, se destinan principalmente para autoconsumo y, en mínima cantidad, para venta (12); por tanto, surge la hipótesis de que la ardilla ( $S$. granatensis) no representa una plaga para los cultivos de maíz, sino que el significado del daño es dado por el impacto sobre la economía campesina, por sus bajos valores de producción de maíz (Z. mays) y de otros cultivos. Lo anterior se apoya en que no existe congruencia entre los reportes de daño por encuestas y cantidad de imágenes captadas de especímenes de ardillas (S. granatensis) en el Valle de Tenza.

Adicionalmente, la cantidad de maíz sano es mucho mayor que la cantidad de maíz dañado por fauna silvestre; esto también se relaciona con valores de densidad poblacional reportados por González et al. (13) para la ardilla (S. granatensis) en cinco municipios del Valle de Tenza, los cuales varían de 0,0 a 57,96 ardillas $/ \mathrm{km}^{2}$, valores similares $\left(\mathrm{D}=7,9\right.$ a 27,6 ardillas $\left./ \mathrm{km}^{2}\right)$ a los reportados por Garcés \& Rodríguez (14) en los Andes colombianos, en diferentes tipos de cobertura.

Se eligieron estrategias de control dirigidas a la mayoría de las especies involucradas, tomando dos métodos referenciados por Del Villar (5) y Lamarque et al. (10). Para el caso del biopreparado con ají, este contiene capsaicina, que es un compuesto irritante en mamíferos que produce sensación de dolor al entrar en contacto con el receptor vallinoide, responsable de la sensibilidad al calor y otros estímulos dolorosos (15); teniendo en cuenta que las aves no son sensibles a este, se aplicó creolina, catalogada como repelente olfativo (5) que aleja 
tanto a mamíferos y aves como a algunos artrópodos que pueden ocasionar daño a cultivos.

Se observó que en las parcelas 2, 6, 7 y 8, rodeadas de parches de bosque, el daño se ubicó en sus bordes, pero en la parcela 5 (testigo) la ardilla ( $S$. granatensis) no dañó ningún fruto y solo se encontraron 12 mazorcas afectadas por el gusano y el gorgojo del maíz; esta parcela estaba rodeada por potrero y un río, por tanto, se asume que las ardillas tienen impedimento para acceder a ella. La parcela 6 (testigo) se encontraba rodeada por bosque, que facilita el acceso de los animales al cultivo, especialmente de las ardillas ( $S$. granatensis), que dependen de los árboles para su movilidad, lo que puede explicar el alto número de maíz dañado en esta parcela. Bucher (16) menciona que debido a la fragmentación de hábitats se encuentran paisajes de mosaico, en los que se alternan parches de bosque con parcelas de cultivos; esto ofrece a la fauna silvestre la disponibilidad del alimento cercano a áreas adecuadas para la supervivencia. La interpretación del tipo de daño en estas parcelas permite sugerir que la cantidad de maíz dañado está relacionado con la cercanía de los cultivos en cada tipo de vegetación adyacente (bosque, potrero o río), independientemente del tipo de tratamiento.

Aunque la cantidad de maíz (Z. mays) dañado es estadísticamente igual entre los tratamientos y el testigo, se aprecia que es mayor la mediana de la cantidad de maíz sano que el dañado en todos los tratamientos frente al testigo $(130,5$ vs. 6,0 respectivamente), independientemente del tratamiento. Estos resultados indican que los tratamientos probados no tienen efecto sobre la protección de los cultivos. La proporción de maíz (Z. mays) dañado es $4,59 \%$ de la producción total en valores promedio; es decir, los agricultores obtienen maíz sano en proporciones altas respecto del dañado. El significado de lo anterior se relaciona con la afirmación de que las poblaciones de ardillas (S. granatensis) y otras de fauna silvestre no serían plaga sobre el maíz (Z. mays).

\section{Conclusiones}

Los agricultores reportan, según las encuestas, daños a cultivos en el Valle de Tenza por cuatro especies de mamíferos, principalmente la ardilla (S. granatensis) y el fara (D. marsupialis), y seis de aves; sin embargo, con los métodos de captura con trampas y fotografías se obtuvieron bajos registros de estas ardillas y faras en cinco sitios de muestreo donde hay cultivos. Existe diferencia altamente significativa $(P<0,01)$ entre el total del maíz dañado y sano ocasionado por la fauna silvestre, sin importar el tipo de tratamiento o testigo, pero no existe diferencia significativa entre el maíz dañado o sano entre los tratamientos $(P>0,05)$. El resultado final es la producción del $95 \%$ de maíz sano respecto del total, por tanto, surge la hipótesis de que las poblaciones de ardilla podrían no ser plaga en los cultivos de maíz.

\section{Agradecimientos}

A Corpochivor y al Grupo GEO de la Universidad Pedagógica y Tecnológica de Colombia por la financiación y acompañamiento durante el desarrollo de la investigación.

\section{Referencias}

(1) Romero-Balderas KG, Naranjo EJ, Morales $\mathrm{HH}$, Nigh RB. Daños ocasionados por vertebrados silvestres al cultivo de maíz en la selva Lacandona, Chiapas, México. Interciencia. 2006; 31(4): 276283.

(2) Elías DJ, Valencia D. La agricultura latinoamericana y los vertebrados plagas. Interciencia. 1984; 9(4): 223-229.

(3) Valencia D., Elías DJ, Ospina JA. Rodent pests in Colombian agriculture. En: Proceedings of the Sixteenth Vertebrate Pest Conference. 1994.

(4) Gómez O., Dredge D. Las ardillas de México. Biodiversitas. 2003; 51: 8-11. 
(5) Del Villar-González, D. Principales vertebrados plaga en México: situación actual y alternativas para su manejo. Rev. Chapingo. 2000; 6(1): 41-54.

(6) Atkinson R., Flint J. Accessing Hidden and Hard to Reach Populations: Snowball Research Strategies. Soc. Res. 2001; 33.

(7) Ministerio de Ambiente, Vivienda y Desarrollo Territorial. Guía de Identificación de Fauna Silvestre Colombiana. 2009 (Acceso 10 de junio de 2014):14. Disponible en: http://cam.gov.co/ sitio/images/documents/phocadownload/guias de_identificacion/guia \% 20identificacion $\% 20$ fauna \% 20silvestre\%20colombiana.pdf.

(8) FAO. Biopreparados para el manejo sostenible de plagas y enfermedades en la agricultura urbana y periurbana. Primera Edición. 2010 (Acceso 01 de julio del 2014). Disponible en: http://www.fao.org/3/a-as435s.pdf.

(9) Statgraphics Plus 5.1. 1990-1995. Microsoft Corp. USA

(10) Lamarque F., Anderson J., Fergusson R., Lagrange M., Osei-Owusu Y., Bakker L. Human-wildlife conflict in Africa. Causes, consequences and management strategies. Food and Agriculture Organization of the United Nations. 2009.

(11) ILSA s/a. Caracterización de la economía campesina en Colombia. 2015 (Acceso 2 de agosto del año
2015). Disponible en: http://ilsa.org.co:81/biblioteca/dwnlds/otras/tlc/cap3.pdf.

(12) CDIM ESAP. Centro de acopio campesino municipio de Guateque, Boyacá. Estudio de mercado y orientaciones generales para la administración del centro de acopio. Características socioeconómicas generales de la provincia del Oriente y del municipio de Guateque. 2001. (Acceso 26 de febrero de 2017), pp. 1-27.

(13) González D., Rodríguez P., Montes R., Castillo I. Densidad de Sciurus granatensis en hábitats intervenidos del Valle de Tenza, Boyacá, Colombia. Rev. Cienc. Nat. Agro. 2015; 2(2): 225-237.

(14) Garcés M., Rodríguez C. Densidad de ardilla roja (Sciurus granatensis) en hábitats con diferentes coberturas vegetales en los Andes de Colombia. Mastozool Neotrop. 2013; 20(2): 381-386.

(15) National Institute of Neurological Disorders and Stroke. Dolor: Esperanza en la Investigación. Instituto Nacional de Trastornos Neurológicos y Accidentes Cerebrovasculares. 2014 (Acceso 31 de julio del año 2014). Disponible en: http://espanol. ninds.nih.gov/trastornos/dolor.htm.

(16) Bucher E. Las Aves como plaga en Argentina. Centro de Zoología Aplicada, Universidad Nacional de Córdoba. 1992. 